\title{
LAS CONCEPCIONES DE LOS PROFESORES DE CIENCIAS SOBRE LOS PROCESOS EDUCATIVOS: REVISIÓN Y PROPUESTAS PARA LA FORMACIÓN INICIAL DEL PROFESORADO DE SECUNDARIA
}

\section{THE CONCEPTIONS OF SCIENCE TEACHERS ABOUT THE EDUCATIONAL PROCESSES: REVISION AND PROPOSALS FOR THE INITIAL SECONDARY TEACHER TRAINING}

\author{
FRANCISCO José POYATO LÓPEZ \& ALFONSO PONTES PEDRAJAS *1 \\ *UNIVERSIDAD DE CÓRDOBA (ESPAÑA)
}

\section{RESUMEN}

Desde hace algún tiempo, estamos trabajando en un proyecto de investigación relacionado con la mejora de la formación inicial del profesorado de secundaria, por considerar que se trata de un tema fundamental para el futuro de nuestro sistema educativo. Uno de los aspectos más relevantes de este proyecto es la exploración de las concepciones previas de los futuros profesores del área científico-técnica y el diseño de propuestas metodológicas que contribuyan a su evolución y progreso durante el proceso de formación inicial. En este contexto nos ha surgido la necesidad de realizar una revisión de trabajos anteriores sobre esta temática, con objeto de proporcionar unos fundamentos sólidos al desarrollo de los trabajos que puedan realizarse en el marco de este proyecto. La finalidad principal de este estudio consiste en mostrar un resumen de la revisión realizada sobre el pensamiento curricular del profesorado, en torno a los procesos de aprendizaje, enseñanza y evaluación de las ciencias.

Palabras clave: Concepciones del profesorado, enseñanza y aprendizaje de las ciencias, evaluación, formación inicial docente.

\begin{abstract}
For some time, we have been working on a research project related to the improvement of the initial training of secondary school teachers, considering that it is a fundamental issue for the future of our educational system. One of the most important aspects of this project is the exploration of the previous conceptions of the future professors of the scientific-technical area and the design of methodological proposals that contribute to their evolution and progress during the initial formation process. In this context, we have had the need to review previous works, in order to provide a solid foundation for the development of the work that can be done within the framework of this project. The main purpose of this study is to show a summary of the review made on the curricular thinking of teachers, around the processes of learning, teaching and evaluation of science.
\end{abstract} training.

Keywords: Teacher conceptions, teaching and learning of sciences, evaluation, initial teacher

\footnotetext{
${ }^{1}$ Autor de correspondencia: Francisco José Poyato López. Email: fpoyato@uco.es
} 


\section{Introducción}

La investigación educativa relacionada con la formación del profesorado de ciencias ha aumentado de forma considerable desde el importante cambio que supuso la implantación del Máster de Enseñanza Secundaria (MAES). Entre las aportaciones relacionadas con esta línea de trabajo, destacan aquellas que toman como punto de partida las ideas previas y opiniones de los futuros docentes sobre todos aquellos temas que están relacionados con su formación pedagógica y didáctica (Campanario, 1998).

La importancia de analizar las concepciones curriculares de los futuros docentes, más aún desde la perspectiva del enfoque reflexivo, está plenamente demostrada (Perrenoud, 2010). La influencia, tanto en la etapa escolar como universitaria, que sus experiencias como alumnos ejercen sobre dichas concepciones y creencias es de enorme peso. En este sentido resulta necesario que durante el periodo de formación inicial del profesorado, además de los contenidos, el alumnado analice sus propias concepciones personales a través de actividades de aula para, de esta manera, poder tomar conciencia del modelo didáctico subyacente tras estas ideas y, desde el análisis reflexivo de este metaconocimiento, poder avanzar en aquellas competencias docentes que permitan asumir modelos didácticos más adecuados con las necesidades del alumnado de la actual educación secundaria (Abell, 2007).

En el contexto de la formación inicial del profesorado, se han llevado a cabo numerosas investigaciones que ponen de manifiesto la necesidad de indagar en las concepciones y creencias sobre los procesos educativos. La investigación que presentamos con este trabajo, tal y como ha ocurrido en anteriores llevados a cabo por otros autores, surge de la necesidad de contar con revisiones bibliográficas adecuadas y actualizadas sobre este tema, que analicen críticamente la información presentada y que faciliten el acceso a la información de investigadores del área para ayudar a fundamentar los trabajos de innovación e investigación sobre esta temática.

La revisión de estudios que hemos realizado se centra en aquellos que se aproximan al pensamiento curricular del profesorado de ciencias (en activo o en formación). Concretamente nos hemos dirigido a aquellos que están orientados hacia la investigación sobre los procesos de aprendizaje, enseñanza y evaluación. De esta manera, un primer apartado está referido a aquellos trabajos que han abordado el estudio de varios procesos educativos simultáneamente, y a continuación, mostramos una exploración de aquellos otros que se han aproximado al análisis por separado de las concepciones o las creencias sobre el proceso de aprendizaje, el proceso de enseñanza o el proceso de evaluación del aprendizaje.

\section{Estudios sobre el pensamiento curricular del profesorado de ciencias recogidos en investigaciones anteriores relevantes}

En este apartado vamos a centrarnos, primer lugar, en sintetizar las ideas procedentes de otras investigaciones clásicas, consideradas como trabajos de referencia, en las que se han realizado revisiones interesantes de estudios sobre las concepciones y creencias del profesorado de ciencias sobre diversos procesos educativos. Posteriormente se analizarán estudios más recientes, que consideramos interesantes, aunque resulta verdaderamente difícil mencionar a todos los autores y autoras que han hecho aportaciones relevantes en esta área de trabajo tan extensa.

\subsection{Sintesis de revisiones previas sobre los procesos educativos}

En la tabla 1 se sintetizan las principales investigaciones revisadas en la primera investigación de referencia consultada para la elaboración de este trabajo (Mellado, 1996). 
Tabla 1.

Referencias más representativas incluidas en la $1^{\text {a }}$ revisión

\begin{tabular}{|c|c|}
\hline Autores (año) & Temática principal: procesos de enseñanza y aprendizaje \\
\hline $\begin{array}{l}\text { Hewson y Hewson } \\
\text { (1989); Gunstone et } \\
\text { al., (1993) }\end{array}$ & $\begin{array}{l}\text { Los años de escolaridad que los profesores de ciencias han pasado como alumnos } \\
\text { tienen una gran influencia en sus concepciones pedagógicas sobre los procesos } \\
\text { educativos }\end{array}$ \\
\hline Aguirre et al. (1990) & $\begin{array}{l}\text { Las creencias pedagógicas son muy estables y apenas cambian durante el programa } \\
\text { de formación inicial }\end{array}$ \\
\hline Kennedy (1991) & $\begin{array}{l}\text { El aprendizaje supone absorción de conocimiento, la enseñanza consiste en contar a } \\
\text { los estudiantes lo que el profesor conoce y evaluar consiste en registrar el recuerdo de } \\
\text { los estudiantes de ese conocimiento }\end{array}$ \\
\hline Autores (año) & Temática principal: aprendizaje de las ciencias \\
\hline Spear (1984) & $\begin{array}{l}\text { Los profesores secundaria ingleses tienen mayores expectativas sobre el potencial } \\
\text { hacia la ciencia de los alumnos que de las alumnas, lo cual condiciona la evaluación. }\end{array}$ \\
\hline Porlán (1989) & $\begin{array}{l}\text { Entre los profesores de primaria en formación predomina el modelo implícito de «mente } \\
\text { en blanco» para el aprendizaje de las ciencias. }\end{array}$ \\
\hline Aguirre et al. (1990) & $\begin{array}{l}\text { La mayoría de los profesores de secundaria en formación inicial tiene una idea del } \\
\text { aprendizaje de la ciencia como una recepción de conocimiento por los alumnos. }\end{array}$ \\
\hline Autores (año) & Temática principal: Enseñanza \\
\hline Porlán (1989) & $\begin{array}{l}\text { Los profesores de primaria en formación rechazan el método tradicional expositivo y } \\
\text { caen en un practicismo espontaneísta. }\end{array}$ \\
\hline Pomeroy (1993) & $\begin{array}{l}\text { Los profesores tienen una concepción más tradicional que las profesoras y los } \\
\text { profesores de secundaria significativamente más tradicionales que los de primaria. }\end{array}$ \\
\hline López-Ruiz (1994) & $\begin{array}{l}\text { Distingue entre los profesores que tienen una concepción «constructivista compleja», } \\
\text { y los que tienen una concepción «constructivista simplificada }\end{array}$ \\
\hline
\end{tabular}

En la tabla 2 se sintetizan las principales investigaciones revisadas en la segunda investigación de referencia consultada para este trabajo (Porlán et al. 1998).

Tabla 2.

Referencias más representativas incluidas en la $2^{\mathrm{a}}$ revisión

\begin{tabular}{|c|c|}
\hline Autores (año) & Temática principal: Enseñanza \\
\hline Elbaz (1981) & $\begin{array}{l}\text { Descripción en profundidad del conocimiento práctico de una profesora, cuyo contenido } \\
\text { hace referencia a cinco categorias. }\end{array}$ \\
\hline $\begin{array}{l}\text { Halkes y Deijkers } \\
\text { (1983) }\end{array}$ & $\begin{array}{l}\text { Ponen de relieve tres núcleos esenciales de concepciones sobre la enseñanza: el } \\
\text { control del profesor, la participación de los estudiantes y el flujo de las actividades. }\end{array}$ \\
\hline Bauch (1984) & $\begin{array}{l}\text { Clasifica a los profesores en dos grupos: uno centrado en el control de la clase y otro } \\
\text { preocupado por la participación de los alumnos. }\end{array}$ \\
\hline Marrero (1994) & $\begin{array}{l}\text { Las concepciones de los profesores participantes se enmarcan en diferentes teorías } \\
\text { sobre la enseñanza. }\end{array}$ \\
\hline Autores (año) & $\begin{array}{c}\text { Temática principal: Relación entre las concepciones científicas y didácticas de los } \\
\text { profesores (E-A) }\end{array}$ \\
\hline $\begin{array}{l}\text { Pope y Scott } \\
\text { (1988) }\end{array}$ & $\begin{array}{l}\text { Los profesores que sostienen la concepción positivista de la ciencia pondrán poco } 0 \\
\text { ninaún énfasis en las concepciones v en la particioación activa }\end{array}$ \\
\hline $\begin{array}{l}\text { Hollon y Anderson } \\
\text { (1987) }\end{array}$ & $\begin{array}{l}\text { Apenas se tienen en cuenta las ideas del alumnado sobre los conceptos de ciencias a la } \\
\text { hora de enseñar }\end{array}$ \\
\hline $\begin{array}{l}\text { Smith y Neale } \\
\text { (1991) }\end{array}$ & $\begin{array}{l}\text { Encontraron en los participantes cuatro tendencias basadas en: el descubrimiento, los } \\
\text { procesos, el dominio del contenido y el cambio conceptual. }\end{array}$ \\
\hline $\begin{array}{l}\text { Gustafson y } \\
\text { Rowell (1995) }\end{array}$ & $\begin{array}{l}\text { En la formación inicial el futuro docente piensa que los alumnos aprenden a partir de } \\
\text { procesos de manipulación, y prácticas y que la ciencia se concibe como un cuerpo de } \\
\text { conocimientos que se adquiere mediante un determinado proceso de búsqueda. }\end{array}$ \\
\hline
\end{tabular}

En la tabla 3 se sintetizan las principales investigaciones revisadas en la tercera investigación de referencia consultada (Martínez Aznar et al., 2001). 
Tabla 3.

Referencias más representativas incluidas en la $3^{\mathrm{a}}$ revisión

\begin{tabular}{|c|c|}
\hline Autores (año) & Temática principal \\
\hline $\begin{array}{l}\text { Porlán (1989); Marrero } \\
\text { (1994); Gallagher (1991) }\end{array}$ & $\begin{array}{l}\text { Estudiando a futuros profesores y a profesores en activo detectó una variedad de } \\
\text { modelos didácticos: Modelo tecnológico, Modelo tradicional y Modelo Alternativo }\end{array}$ \\
\hline Porlán et al. $(1997,1998)$ & Las posturas tradicionales son predominantes \\
\hline $\begin{array}{l}\text { Pérez Gómez y Gimeno } \\
\text { (1992) }\end{array}$ & $\begin{array}{l}\text { Concepciones abiertas en cuanto a metodología y contenidos, pero con } \\
\text { tendencias tradicionales en cuanto a evaluación }\end{array}$ \\
\hline $\begin{array}{l}\text { Hollon, Roth y Anderson } \\
\text { (1991) }\end{array}$ & $\begin{array}{l}\text { Incoherencias entre selección de contenidos y propuestas metodológicas a } \\
\text { aplicar }\end{array}$ \\
\hline $\begin{array}{l}\text { Gustafson y Rowell } \\
\text { (1995); Hashweh (1996) }\end{array}$ & $\begin{array}{l}\text { Las ideas de los alumnos y su participación en la tarea docente no son tenidas en } \\
\text { cuenta. }\end{array}$ \\
\hline $\begin{array}{l}\text { Furió et al. (1992); } \\
\text { Mellado (1998) }\end{array}$ & $\begin{array}{l}\text { Los profesores manifiestan un cierto tipo de conocimiento para actuar adquirido } \\
\text { por formación ambiental durante la escolaridad, que tiene una gran influencia en } \\
\text { la actuación futura y que no suele alterarse con la formación inicial }\end{array}$ \\
\hline $\begin{array}{l}\text { Fernández y Elortegui } \\
\text { (1996) }\end{array}$ & $\begin{array}{l}\text { Modelos: transmisor o tradicional, tecnológico, artesano, descubridor y } \\
\text { constructor. }\end{array}$ \\
\hline
\end{tabular}

En la tabla 4 se sintetizan las principales investigaciones revisadas en la cuarta investigación de referencia (Marín y Benarroch, 2010).

Tabla 4.

Referencias más representativas incluidas en la $4^{\mathrm{a}}$ revisión

\begin{tabular}{|c|c|}
\hline Autores (año) & Temática principal \\
\hline $\begin{array}{l}\text { Hollon y Anderson } \\
\text { (1987); Porlán (1989); } \\
\text { Hewson y Hollon (1994) }\end{array}$ & $\begin{array}{l}\text { Los alumnos aprenden si están atentos a la explicación del profesor y estudian. } \\
\text { Ese aprendizaje se manifiesta como lo que son capaces de explicar en los } \\
\text { exámenes. Hay que cambiar las ideas de los alumnos por la «verdad científica». }\end{array}$ \\
\hline Huibretse et al. (1994) & $\begin{array}{l}\text { La práctica docente parece matizada por el conjunto de valores, sentimientos, } \\
\text { pensamientos y acciones formadas durante la etapa como estudiantes }\end{array}$ \\
\hline Dillon et al., (1994) & $\begin{array}{l}\text { Relacionan entre las creencias del profesorado sobre la enseñanza y aprendizaje } \\
\text { de las ciencias con su conducta en el aula al enseñar ciencias }\end{array}$ \\
\hline $\begin{array}{l}\text { Desaultes (1993); López } \\
\text { (1995) }\end{array}$ & $\begin{array}{l}\text { Los profesores no poseen siempre concepciones uniformes y coherentes sobre la } \\
\text { enseñanza y el aprendizaje de sus alumnos. }\end{array}$ \\
\hline Mellado (1996) & $\begin{array}{l}\text { Revisa estudios que indican que las concepciones constructivistas del } \\
\text { profesorado no son demasiado coherentes o uniformes }\end{array}$ \\
\hline Porlán et al. (1997; 1998) & $\begin{array}{l}\text { Encuentran posiciones mayoritarias próximas al enfoque tradicional y otras } \\
\text { próximas al enfoque constructivista (pero menos extendidas) }\end{array}$ \\
\hline $\begin{array}{l}\text { Baena (2000); Boulton- } \\
\text { Lewis y Wills (2001) }\end{array}$ & $\begin{array}{l}\text { Existe mayor coherencia entre enseñanza y aprendizaje cuando se mantienen } \\
\text { posiciones más tradicionales que cuando están más cercanas al constructivismo }\end{array}$ \\
\hline $\begin{array}{l}\text { Porlán y Martín del Pozo } \\
\text { (2004) }\end{array}$ & $\begin{array}{l}\text { Existen varias tendencias sobre cómo se ve el proceso de enseñanza/ } \\
\text { aprendizaje, desde una visión basada en la transmisión-recepción de } \\
\text { conocimiento hasta una visión constructivista minoritaria }\end{array}$ \\
\hline Pozo et al. (2006) & $\begin{array}{l}\text { Analizan las creencias del profesorado sobre enseñanza y aprendizaje como } \\
\text { teorías implícitas }\end{array}$ \\
\hline
\end{tabular}

\section{Revisión de estudios más recientes sobre el pensamiento docente en torno a la educación científica}

Los primeros trabajos sobre las concepciones del profesorado acerca de los procesos de enseñanza y aprendizaje de las ciencias experimentales provienen del mundo anglosajón (Hewson y Hewson, 1989; Aguirre, Haggerty y Linder, 1990) y posteriormente se han desarrollado múltiples estudios sobre esta temática en el ámbito iberoamericano, tanto en la formación de futuros maestros de enseñanza primaria (Porlán, Rivero y Martín, 1997, 1998), como en la formación inicial de profesores 
de secundaria (Fernández, Elortegui y Medina, 2002), de profesores de primaria o secundaria en ejercicio (Martínez-Aznar et al., 2001; Ravanal y Quintanilla, 2012) o de profesores universitarios de ciencias (Martínez y González, 2014). A continuación, mostramos en la tabla 6 algunos de los trabajos más representativos que se han llevado a cabo en los últimos tiempos clasificados en función de los procesos educativos objeto de investigación.

Tabla 6.

Estudios de especial interés sobre el pensamiento docente acerca de los procesos educativos

\begin{tabular}{|c|c|}
\hline Autores (año) & Comentarios sobre aportaciones \\
\hline Solís (2005) & $\begin{array}{l}\text { Existen diferencias en las concepciones sobre la enseñanza, el aprendizaje y la } \\
\text { evaluación de alumnado del CAP entre lo declarado y lo realizado. }\end{array}$ \\
\hline Gullberg et al. (2008) & $\begin{array}{l}\text { Existencia de una gran diversidad en el pensamiento docente del profesorado de } \\
\text { secundaria sobre el aprendizaje y la enseñanza de la ciencia }\end{array}$ \\
\hline Fernández et al. (2009) & $\begin{array}{l}\text { Existencia de incongruencias en las declaraciones sobre la enseñanza y el } \\
\text { aprendizaje que hace el profesorado y sus prácticas educativas. }\end{array}$ \\
\hline Cheng et al. (2009) & $\begin{array}{l}\text { El aprendizaje es un producto del esfuerzo más que de habilidades innatas. En lo } \\
\text { que se refiere a la enseñanza, concluyen que coexisten varios modelos didácticos. }\end{array}$ \\
\hline Contreras, 2010 & $\begin{array}{l}\text { Existe cierta identificación del profesorado con posiciones constructivistas pero } \\
\text { esta identificación no se plasma en la práctica educativa. }\end{array}$ \\
\hline Cifuentes et al. (2010) & $\begin{array}{l}\text { Existe cierta evolución en las concepciones sobre la enseñanza y el aprendizaje de } \\
\text { parte de los maestros y maestras a lo largo de sus tres años de formación inicial }\end{array}$ \\
\hline $\begin{array}{l}\text { Martínez y Benarroch, } \\
2012\end{array}$ & $\begin{array}{l}\text { Son más abundantes las concepciones empiristas frente a las constructivistas } \\
\text { sobre la naturaleza de la ciencia. }\end{array}$ \\
\hline $\begin{array}{l}\text { Rodríguez-Arteche y } \\
\text { Martínez-Aznar (2016) }\end{array}$ & $\begin{array}{l}\text { Dificultad a la hora de asumir metodologías cercanas al enfoque indagativo por } \\
\text { parte del futuro profesorado. }\end{array}$ \\
\hline Rivero et al. (2017) & $\begin{array}{l}\text { Muestran el cambio en el conocimiento acerca de la enseñanza de las ciencias de } \\
\text { maestros y maestras en formación }\end{array}$ \\
\hline Melo et al. (2017) & Evolución en el conocimiento del currículo o la enseñanza. \\
\hline $\begin{array}{l}\text { Pérez, Serrano y Pontes } \\
\text { (2019) }\end{array}$ & $\begin{array}{l}\text { Existe una relación importante entre la visión global de la identidad profesional y el } \\
\text { desarrollo de habilidades educativas. }\end{array}$ \\
\hline
\end{tabular}

\subsection{Revisión de estudios recientes sobre el aprendizaje}

En este apartado incluiremos las investigaciones más destacadas publicadas en los últimos tiempos sobre las concepciones y creencias del aprendizaje y que mostramos a continuación en la tabla 7.

Tabla 7.

Estudios específicos centrados en la visión de los procesos de aprendizaje

\begin{tabular}{|c|c|}
\hline Autores (año) & Temática abordada \\
\hline $\begin{array}{l}\text { Marín y Benarroch } \\
\text { (2009) }\end{array}$ & $\begin{array}{l}\text { Importancia de trabajar con el cuestionario COMVdA para conocer las ideas previas } \\
\text { sobre el aprendizaje de la ciencia y su evolución. }\end{array}$ \\
\hline Meirink et al. (2009) & $\begin{array}{l}\text { Investigan la evolución de las creencias acerca del aprendizaje y la enseñanza de } \\
\text { un grupo de profesores de secundaria }\end{array}$ \\
\hline $\begin{array}{l}\text { Marín y Benarroch } \\
(2010)\end{array}$ & $\begin{array}{l}\text { Aplican un cuestionario de opción múltiple para conocer las ideas previas sobre el } \\
\text { aprendizaje de la ciencia y observar su evolución }\end{array}$ \\
\hline $\begin{array}{l}\text { Virtanen y Lindblom } \\
(2010)\end{array}$ & $\begin{array}{l}\text { Existe una brecha sustancial en la visión del aprendizaje que muestran los } \\
\text { profesores y los alumnos }\end{array}$ \\
\hline Tigchelaar et al. (2012) & Existen diferentes patrones de evolución en las concepciones sobre el aprendizaje \\
\hline $\begin{array}{l}\text { Ravanal y Quintanilla } \\
(2012)\end{array}$ & $\begin{array}{l}\text { Creencias relativamente persistentes y, en ocasiones, contradictorias sobre el } \\
\text { aprendizaje de la biología }\end{array}$ \\
\hline Solís et al. (2013) & $\begin{array}{l}\text { Escasa evolución las concepciones curriculares de los estudiantes de ciencias del } \\
\text { máster de profesorado de secundaria. }\end{array}$ \\
\hline $\begin{array}{l}\text { Martínez y González } \\
\text { (2014) }\end{array}$ & $\begin{array}{l}\text { diversos grados de implantación del enfoque constructivista sobre el aprendizaje en } \\
\text { el alumnado del Máster }\end{array}$ \\
\hline Abril et al. (2014) & Estudian las creencias del profesorado en formación inicial y en activo sobre el \\
\hline
\end{tabular}


modelo de aprendizaje por investigación, encontrando diferencias interesantes entre ambos grupos.

Lin, Lee y Tsai (2014) Patrones de pensamiento próximos a los enfoques educativos tradicional y constructivista en profesores y alumnos de secundaria.

Yakar y Turgut (2017) Se produjo una evolución de las creencias del profesorado en formación hacia visiones más centradas en el alumnado

\subsection{Revisión de estudios relacionados con la visión de profesores de ciencias sobre la enseñanza}

En los últimos tiempos se aprecia un mayor interés por desarrollar estudios que permitan conocer 0 representar la evolución de las concepciones sobre la enseñanza de las ciencias en conexión con los llamados "modelos didácticos" descritos por Porlán et al. (1997) durante el proceso de formación inicial de los docentes. En este contexto, hablar de modelos didácticos implica pensar en un modelo de pensamiento y acción deseable, en un modelo más tradicional o transmisivo y en una serie de modelos intermedios situados entre ambos. En la tabla 8 se sintetizan algunos trabajos relacionados con las visiones sobre la enseñanza.

Tabla 8.

Estudios específicos centrados en la visión de la enseñanza

\begin{tabular}{|c|c|}
\hline Autores (año) & Síntesis de aportaciones \\
\hline $\begin{array}{l}\text { Marshall et al. } \\
(2010)\end{array}$ & $\begin{array}{l}\text { Comprobaron las reservas del profesorado a la hora de adoptar metodologías de } \\
\text { enseñanzas más innovadoras, en relación a la enseñanza de las matemáticas. }\end{array}$ \\
\hline Ladachart (2011) & $\begin{array}{l}\text { Existe coherencia entre las concepciones sobre la enseñanza del profesorado y su } \\
\text { práctica educativa }\end{array}$ \\
\hline Rivero et al. (2011), & $\begin{array}{l}\text { Se aprecian diferentes itinerarios de cambio en la progresión de las concepciones sobre } \\
\text { la metodología de enseñanza y la presencia de dos importantes obstáculos en esta } \\
\text { progresión }\end{array}$ \\
\hline Cortés et al. (2012) & $\begin{array}{l}\text { El alumnado en formación inicial la poca aplicación que le conceden a lo aprendido en } \\
\text { las aulas universitarias. }\end{array}$ \\
\hline $\begin{array}{l}\text { Subramaniam } \\
(2013 ; 2014)\end{array}$ & $\begin{array}{l}\text { Encontró tres formas de concebir los procesos educativos: (1) la enseñanza de la } \\
\text { biología es un proceso interactivo, (2) está basado en la lectura o acceso a la } \\
\text { información y (3) es un proceso visual. }\end{array}$ \\
\hline $\begin{array}{l}\text { García-Carmona y } \\
\text { Cruz-Guzmán } \\
\text { (2016) }\end{array}$ & $\begin{array}{l}\text { Para los participantes en el estudio el dominio del contenido disciplinar y la promoción de } \\
\text { trabajos prácticos son las competencias docentes primordiales para enseñar ciencia. }\end{array}$ \\
\hline Buldur, S. (2017) & $\begin{array}{l}\text { Se produjo una evolución en las creencias sobre la enseñanza de los profesores en } \\
\text { formación inicial durante su periodo formativo. }\end{array}$ \\
\hline $\begin{array}{l}\text { Rodríguez Arteche } \\
\text { y Martínez-Aznar } \\
(2018)\end{array}$ & $\begin{array}{l}\text { Se refleja una evolución significativa hacia una tendencia constructivista, si bien se } \\
\text { observan planteamientos algo más «conservadores» que en las creencias declaradas. }\end{array}$ \\
\hline $\begin{array}{l}\text { Serrano, Pontes y } \\
\text { Pérez (2019) }\end{array}$ & $\begin{array}{l}\text { Las visiones tradicionales de la enseñanza son persistentes en las concepciones del } \\
\text { profesorado en formación inicial. }\end{array}$ \\
\hline
\end{tabular}

\subsection{Revisión de estudios relacionados con la visión del profesorado sobre la evaluación del aprendizaje}

En este vamos a indicar aquellos trabajos más representativos sobre las visiones de la evaluación llevados a cabo en los últimos años. En la tabla 9 se muestran estudios relacionados con la evaluación del aprendizaje. 
Tabla 9.

Estudios específicos centrados en la visión de la evaluación

\begin{tabular}{|c|c|}
\hline Autores (año) & Síntesis de aportaciones \\
\hline $\begin{array}{l}\text { Buendía et al. } \\
\text { (1999) }\end{array}$ & $\begin{array}{l}\text { Las respuestas del profesorado a las cuestiones planteadas se pueden considerar } \\
\text { bastante próximas al enfoque educativo tradicional }\end{array}$ \\
\hline $\begin{array}{l}\text { Borko, (1997); } \\
\text { Prieto y Contreras, } \\
\text { (2008). }\end{array}$ & $\begin{array}{l}\text { Es importante en el proceso de evaluación del aprendizaje el papel que desempeñan } \\
\text { las concepciones, creencias y teorías implícitas del profesorado respecto de dicho } \\
\text { proceso }\end{array}$ \\
\hline $\begin{array}{l}\text { Dixon y Haigh } \\
\text { (2009) }\end{array}$ & $\begin{array}{l}\text { Las creencias de los docentes sobre el proceso de evaluación tienen una enorme } \\
\text { influencia en dicho proceso }\end{array}$ \\
\hline Wang et al. (2010) & $\begin{array}{l}\text { S docentes en formación tienen ideas sobre el modo de evaluar más próximas a } \\
\text { siones tradicionales que aquellos docentes que se encuentran en ejercicio. }\end{array}$ \\
\hline $\begin{array}{l}\text { Segers y Tillema } \\
\text { (2011) }\end{array}$ & $\begin{array}{l}\text { Observaron que los alumnos no distinguen entre evaluación sumativa y formativa, } \\
\text { pero los profesores sí hacen esa distinción. Asimismo, observaron que el profesorado } \\
\text { reconoce los efectos que la evaluación tiene en el proceso de aprendizaje. }\end{array}$ \\
\hline Remesal (2011) & $\begin{array}{l}\text { Existe dificultad para poner en práctica un sistema de evaluación innovador y } \\
\text { alternativo que ayude a mejorar el proceso de aprendiza. }\end{array}$ \\
\hline $\begin{array}{l}\text { Turpo-Gebera } \\
\text { (2011) }\end{array}$ & $\begin{array}{l}\text { En los procesos de formación y capacitación docente, subsisten intervenciones } \\
\text { tradicionales en las formas y modos de evaluar los aprendizajes, así como } \\
\text { posicionamientos relativamente innovadores sobre la evaluación, pero en conjunto } \\
\text { revelan situaciones de incertidumbre y ambivalencia. }\end{array}$ \\
\hline Brown et al. (2011) & $\begin{array}{l}\text { Los maestros de primaria están más de acuerdo que los profesores de secundaria } \\
\text { con la idea de que el sistema de evaluación usado por cada profesor determina la } \\
\text { calidad del proceso de enseñanza y la mejora del aprendizaje. }\end{array}$ \\
\hline Halinen et al. (2014) & $\begin{array}{l}\text { Identificaron tres categorías o modelos docentes de evaluación. Tales autores } \\
\text { consideran que la formación pedagógica, la relación con los compañeros, el apoyo } \\
\text { institucional y la interacción con la comunidad educativa influyen de diferente manera } \\
\text { en el sistema de evaluación que cada docente realiza en la práctica. }\end{array}$ \\
\hline $\begin{array}{l}\text { Monteagudo e } \\
\text { (2015) }\end{array}$ & $\begin{array}{l}\text { Los docentes en ejercicio muestran escasa formación en el ámbito evaluativo y } \\
\text { continúan evaluando al alumnado básicamente mediante procedimientos } \\
\text { tradicionales, como los exámenes escritos que enfatizan contenidos conceptuales. }\end{array}$ \\
\hline
\end{tabular}

Estos resultados ponen de manifiesto que los profesores en formación y en activo tienen ideas diferentes sobre la forma de evaluar y que éstas están relacionadas con su propia visión acerca de la enseñanza y el aprendizaje. En los profesores en activo influye mucho su experiencia docente y el contexto educativo donde actúan, mientras que en los profesores en formación las concepciones previas parecen estar más influenciadas por la forma cómo han sido evaluados como alumnos.

\section{CONCLUSIONES}

El punto de partida de la revisión bibliográfica que hemos realizado se encuentra en los trabajos de otros autores y autoras considerados como relevantes, como son los de Mellado (1996), Porlán et al. (1998), Martínez Aznar et al. (2001) o Marín y Benarroch (2010). En estas investigaciones se ha destacado que las creencias y concepciones de la mayoría del profesorado de la época se relacionan con las posiciones tradicionales sobre la enseñanza y el aprendizaje, destacando también la gran influencia de los años de escolaridad en sus concepciones pedagógicas. Aun así, en las dos últimas referencias citadas, se denota que la relación con estas posiciones tradicionales no es tan marcada como en la década anterior.

Desde la perspectiva desarrollada con la revisión de trabajos sobre las concepciones y creencias que hemos llevado a cabo, podemos extraer varias conclusiones. En primer lugar, existe una gran 
diversidad en el pensamiento docente del profesorado de secundaria sobre el aprendizaje y la enseñanza de la ciencia y al mismo tiempo existen también diferencias en las concepciones sobre la enseñanza, el aprendizaje y la evaluación entre lo declarado y lo realizado. Con respecto a la formación inicial del profesorado, se aprecia cierta evolución en las concepciones sobre la enseñanza y el aprendizaje a lo largo de este periodo formativo.

Las investigaciones sobre creencias y concepciones sobre el aprendizaje, muestran que las creencias iniciales relacionadas con visiones más tradicionales son persistentes, pero en otros casos las creencias expresadas son contradictorias y, por tanto, no se puede hablar de verdadera evolución en sus creencias. En general, se puede decir que existen distintos patrones de evolución. Por otro lado, se aprecian diferentes grados de implantación del enfoque constructivista. En este sentido resulta oportuno incidir en la importancia que cobra la utilización de planteamientos metodológicos como los que hemos señalado anteriormente, y que nos sirven de guía, durante la formación inicial del profesorado,

Las principales conclusiones sobre la revisión de trabajos enfocados en las concepciones 0 creencias sobre la enseñanza concepciones o creencias sobre la enseñanza, nos permiten hablar de cierto escepticismo inconsciente a la hora de adoptar y llevar a la práctica metodologías de enseñanzas más innovadoras por parte del profesorado en formación inicial, hecho este que concuerda con la persistencia de las ideas previas observadas en muchos casos y la dificultad que supone fomentar la evolución de las mismas. También nos tiene que hacer pensar sobre la adopción de estrategias formativas más eficientes o más motivadoras por parte del profesorado.

La revisión de los trabajos que tienen que ver con las concepciones o creencias sobre la evaluación han puesto de manifiesto que en general dichas ideas se pueden considerar bastante próximas al enfoque educativo tradicional. Con todo, los profesores son conscientes de la importancia de la evaluación para mejorar los procesos de enseñanza-aprendizaje, así como para hacer un seguimiento del proceso de construcción del conocimiento del alumnado, pero encuentran que existe dificultad para poner en práctica un sistema de evaluación innovador y alternativo que ayude a mejorar el proceso de aprendizaje. De esta manera, podemos extraer que en el profesorado en formación subsisten maneras tradicionales de concebir el proceso de evaluación del aprendizaje, al igual que posicionamientos relativamente innovadores sobre la evaluación, pero en conjunto revelan situaciones de incertidumbre y ambivalencia y en cualquier caso más próximas a visiones tradicionales.

Esta revisión de los trabajos realizados en los últimos años nos ha permitido apreciar cierta movilización en las creencias y concepciones sobre los procesos educativos en el profesorado en formación inicial. Así y todo, se constata que las ideas de los profesores en formación inicial están más próximas a las metodologías tradicionales, incluso después de la implantación del MAES. Este hecho debería hacer reflexionar sobre cómo se está llevando a cabo esta formación y si no se seguirán repitiendo aquellos errores que numerosas investigaciones habían puesto de manifiesto sobre aspectos cruciales relacionados con la preparación para el ejercicio de la profesión docente.

Tal y como se comentaba al comienzo de este trabajo, consideramos oportuna la revisión bibliográfica que hemos realizado porque contribuye al análisis de la situación actual sobre la investigación que se lleva a cabo actualmente con relación a las concepciones y creencias sobre los procesos educativos de aprendizaje, enseñanza y evaluación. Con ella también hemos contribuido a analizar la evolución que se ha producido en los últimos años sobre la investigación en esta área de conocimiento. Por otro lado, también pretendemos que las aportaciones que hemos hecho sirvan de ayuda para posteriores investigaciones que se aborden sobre esta materia.

\section{Referencias}

Abell, S. K. (2007). Research on Science Teacher Knowledge. En Abell y Lederman (eds.). Handbook of Research on Science Education (1105-1149). Londres: Lawrence Erlbaum Associates, Publishers. 
Abril, A.M., Ariza, M., Quesada, A. y García, F.J. (2014). Creencias del profesorado en ejercicio y en formación sobre el aprendizaje por investigación. Revista Eureka sobre Enseñanza y Divulgación de las Ciencias, 11(1), 22-33.

Aguirre, M. y Haggerty, S. y Linder, C. (1990). Student-teachers' conceptions of science teaching and learning: a case study in preservice science education. International Journal of Science Education, 12(4), 381-390.

Bauch, P.A. (1984). The Impact of Teachers' instructional Beliefs on their Teaching: Implications for Research and Practice. Reunión anual de la AERA. New Orleans.

Borko, H. (1997). New Forms of Classroom Assessment: Implications for Staff Development. Theory into Practice, 36, 231-238.

Boulton-Lewis, G.M. y Wilss, L. (2001). Changes in conceptions of learning for Indigenous Australian university students. British Journal of Educational Psychology, 71, 327-341.

Brown, G. T. L., Lake, R., \& Matters, G. (2011). New Zealand and Queensland teachers' conceptions of curriculum: Potential jurisdictional effects of curriculum policy and implementation. Curriculum Perspectives, 31(3), 33-48.

Buendía, L., Carmona, M., González, D. y López, R. (1999), Concepciones de los profesores de educación secundaria sobre evaluación. Educación XXI, 2, 125-154.

Buldur, S. (2017). A Longitudinal Investigation of the Preservice Science Teachers' Beliefs about Science Teaching during a Science Teacher Training Programme. International Journal of Science Education, 39(1), 1-19.

Campanario, J. (1998). ¿Quiénes son, qué piensan y que saben los futuros maestros y profesores de Ciencias? Una revisión de estudios recientes. Revista Interuniversitaria de Formación del Profesorado, 33, 121-140.

Cheng, M. Chan, K-W., Tang, S. y Cheng, A. (2009). Pre-service teacher education students' epistemological beliefs and their conceptions of teaching. Teaching and Teacher Education, 25 (2), 319-327.

Cifuentes, M., Muñoz, J., Santamaría, R. (2010). La evolución del pensamiento de los alumnos de magisterio en algunas cuestiones clave de su formación inicial. Revista Interuniversitaria de Formación del Profesorado, 69, 167-186

Contreras, A. (2010). Las creencias y actuaciones curriculares de los profesores de ciencias de Secundaria de Chile (Tesis Doctoral). Universidad Complutense de Madrid.

Desaultes, S. (1993). La formation à l'enseignement des sciences: le virage épistemologique. Didaskalia, 1, 49-67.

Dillon, D.R., O'Brien, D.G., Moje, E.B. y Stewart, R.A. (1994). Literacy learning in secondary school science classrooms: A cross-case analysis of three qualitative studies. Journal of Research in Science Teaching, 31(4), 345-362.

Dixon, H., y Haigh, M. (2009). Changing mathematics teachers' conceptions of assessment and feedback. Teacher Development, 13(2), 173-186.

Elbaz, F. (1981). The Teachers' Practical Knowledge: Reportof a Case Study. Curriculum Inquiry, 11(1), 43-71.

Fernández G., Elórtegui, N. J. y Medina, M (2002). La formación del profesorado de Ciencias de la Naturaleza de Secundaria, a partir de sus ideas previas. Investigación en la Escuela, 47, 65-74.

Fernández, J. y Elórtegui, N. (1996). Qué piensan los profesores acerca de cómo se debe enseñar. Enseñanza de las Ciencias, 14(3), 331-342.

Fernández, M.T., Tuset, A.M., Pérez, R. y Leyva, A. (2009). Concepciones de los maestros sobre la enseñanza y el aprendizaje y sus prácticas educativas en clases de ciencias naturales. Enseñanza de las Ciencias, 27(2), 287-298.

Furió, C., Gil, D., Pessoa, A.M. y Salcedo, L.E. (1992). La formación inicial del profesorado de educación secundaria. Investigación en la Escuela, 16, 7-21. 
Gallagher, J.J. (1991). Prospective and practicing secondary school science teachers' knowledge and belief about the philosophy of science. Science Education ,75(1), 121-133.

Gullberg, A. Kellner, E Attorps, I. Thoren, I. y Tarneberg, R. (2008). Prospective teachers' initial conceptions about pupils' understanding of science and mathematics. European Journal of Teacher Education, 31(3), 257-278.

Gunstone, R.F., Slattery, M., Bair, J.R. y Northfield, J.R. (1993). A case study exploration of development in preservice science teachers. Science Education, 77(1), 47-73.

Gustafson, B.J. y Rowell, P.M. (1995). Elementary preservice teachers: constructing conceptions about learning science, teaching science and the nature of science. International Journal of Science Education, 17(5), 589-605.

Halinen, K., Ruohoniemi, M., Katajavuori, N. y Virtanen, V. (2014). Life Science Teachers' Discourse on Assessment: A Valuable Insight into the Variable Conceptions of Assessment in Higher Education. Journal of Biological Education, 48(1), 16-22.

Halkes, R. y Deijkres, R. (1983). Teachers' Teaching Criteria, en Halkes, R. y Olson, J.K. Teacher Thinking: a New Perspective on Persisting Problems in Education. Lisse: Swets y Zeitlinger.

Hashweh, M.Z. (1996). Effects of Science Teachers' Epistemological Beliefs in Teaching. Journal of Research in Science Teaching, 33(1), 47-63.

Hewson, P.W. y Hewson, M. G. (1989). Analysis and use of a task for identifyng conceptions of teaching science. Journal of Education for Teaching, 15(3), 191-209.

Hewson, P.W. y Hollon, R. (1994). Connecting thought and action in high school science classrooms. Wisconsin- Madison: Wisconsin Center for Education Research.

Hollon, R. y Anderson, C. (1987). Teacher's beliefs about student's learning processes in science: Selfreinforcing beliefs sistems. Artículo presentado en The Annual Meeting of A.E.R.A. Washington D.C.

Hollon, R.E., Roth, K.J. y Anderson, C.W. (1991). Science teachers' conceptions of teaching and learning. Advances in Research on Teaching, 2, 145-185.

Huibretse, I., Korthagen orthagen, F. y Wubbels, T. (1994). Physics teachers' conceptions of learning, teaching and professional development. International Journal of Science Education, 16(5), 539561.

Kennedy, M. (1991). An Agenda for Research on Teacher Learning. Office of Educational Research and Improvement (ED), Washington, DC.

Lin, T. Lee, M. y Tsai, C. (2014). The commonalities and dissonances between high- school students' and their science teachers' conceptions of science learning and conceptions of science assessment: a Taiwanese sample study. International Journal of Science Education. 36(3), 382405.

López, J.I. (1994). El pensamiento del profesor sobre el conocimiento de los alumnos. Investigación en la Escuela, 22, 58-66.

Marín, N. y Benarroch, A. (2009). Desarrollo, validación y evaluación de un cuestionario de opciones múltiples para identificar y caracterizar las visiones sobre la naturaleza de la ciencia de profesores en formación. Enseñanza de las Ciencias, 27(1), 89-108.

Marín, N. y Benarroch, A. (2010). Cuestionario de opciones múltiples para evaluar creencias sobre el aprendizaje de las ciencias. Enseñanza de las Ciencias, 28(2), 245-260.

Marrero, J. (1994). Las teorías implícitas del profesorado: vínculo entre la cultura y la práctica de la enseñanza. En Rodrigo, M.J., Rodríguez, A. y Marrero, J. (eds.). Las teorías implícitas. Una aproximación al conocimiento cotidiano (PP. 243-270). Madrid: Visor.

Martínez Aznar, M.M., Martín del Pozo, R., Rodrigo Vega, M., Varela Nieto, M.P., Fernández Lozano, M.P., y Guerrero Serón, A. (2001) ¿Qué pensamiento profesional y curricular tienen los futuros profesores de ciencias de secundaria? Enseñanza de las Ciencias, 19(1), 67-87. 
Martínez, C. y González, C. (2014). Concepciones del profesorado universitario acerca de la ciencia y su aprendizaje y cómo abordan la promoción de competencias científicas en la formación de futuros profesores de Biología. Enseñanza de las Ciencias, 32(1), 51-81.

Martínez, M. y Benarroch, A. (2012). Concepciones y creencias sobre ciencia, aprendizaje y enseñanza de profesores universitarios de ciencias. Revista Electrónica de Investigación en Educación en Ciencias, 8(1), 24-41.

Meirink, J., Meijer, P., Verloop, N. y Bergen, T. (2009). Understanding teacher learning in secondary education: the relations of teacher activities to changed beliefs about teaching and learning. Teaching and Teacher Education, 25(1), 89-100.

Mellado, V. (1996). Concepciones y prácticas de aula de profesores de ciencias, en formación inicial, de primaria y secundaria. Enseñanza de las Ciencias, 14(3), pp. 398-402.

Mellado, V. (1998). El estudio de aula en la formación continua del profesorado de ciencias. Alambique, 15 , pp. 39-46.

Melo, L. Cañada, F. y Díaz, M. (2017). Formación continua del profesorado de Física a través del conocimiento didáctico del contenido sobre el campo eléctrico en Bachillerato: un caso de estudio. Caderno Brasileiro de Ensino de Física, 34(1), 131-151.

Monteagudo Fernández, J., Molina Puche, S. y Miralles Martínez, P. (2015). Opiniones sobre evaluación de los profesores de segundo ciclo de ESO de Geografía e Historia en España: El caso de la Región de Murcia. Revista Mexicana de Investigación Educativa, 20(66), 737-761.

Pérez Gómez, A. I. y Gimeno, J. (1992). El pensamiento pedagógico de los profesores: un estudio empírico sobre la incidencia de los cursos de aptitud pedagógica (CAP) y de la experiencia profesional en el pensamiento de los profesores. Investigación en la Escuela, 17, 51-73.

Pérez, E., Serrano, R. y Pontes, A. (2019). Analysis of Science and Technology pre-service teachers' beliefs on the construction of the Teachers' Professional Identity during the initial training process. Eurasia Journal of Mathematics, Science and Technology Education, 15(10), 1-14.

Perrenoud, P. (2010). Desarrollar la práctica reflexiva en el oficio de enseñar: profesionalización y razón pedagógica. Barcelona: Graó.

Pomeroy (1993). Implications of teachers' beliefs about the nature of science: Comparison of the beliefs of scientist, secondary science teachers and elementary teachers. Science Education, 77(3), 261-278.

Pope, M. y Scott, E.M. (1988). La epistemología y la práctica de los profesores, en Porlán, R., García, J.E. y Canal, P. (eds.). Constructivismo y enseñanza de las ciencias (179-191). Sevilla: Díada.

Porlán, R. (1989). Teoría del conocimiento, teoría de la enseñanza y desarrollo profesional. Las concepciones epistemológicas de los profesores (Tesis doctoral). Universidad de Sevilla.

Porlán, R. y Martín del Pozo, R. (2004). The conceptions of in-service and prospective primary school teachers about the teaching and learning of science. Journal of Science Teacher Education, 15(1), 39-62.

Porlán, R., Rivero, A. y Martín del Pozo, R. (1997). Conocimiento profesional y epistemológico de los profesores I: Teoría, métodos e instrumentos. Enseñanza de las Ciencias, 15(2), 155-171.

Porlán, R., Rivero, A. y Martín del Pozo, R. (1998). Conocimiento profesional y epistemología de los profesores II: Estudios empíricos y conclusiones. Enseñanza de las Ciencias, 16(2), 271-288.

Pozo, J.I., Scheuer, N., Pérez Echeverría, M. P., Mateos, M. y De la Cruz, M. (2006). Nuevas formas de pensar la enseñanza y el aprendizaje. Barcelona: Graó.

Prieto, M. y Contreras, G. (2008). Las concepciones que orientan las prácticas evaluativas de los profesores: un problema a develar. Revista Estudios Pedagógicos, 34(2), 245- 262.

Ravanal, L.E. y Quintanilla, M. (2012). Concepciones del profesorado de biología en ejercicio sobre el aprendizaje científico escolar. Enseñanza de las ciencias, 30(2), 33-54.

Remesal, A. (2011). Primary and secondary teachers' conceptions of assessment: A qualitative study. Journal of Teaching and Teacher Education, 27(2), 472-482. 
Rivero, A., Martín del Pozo, R., Solís, E., Azcárate, P., Porlán, R. (2017). Cambio del conocimiento sobre la enseñanza de las ciencias de futuros maestros. Enseñanza de las Ciencias, 35(1), 2952.

Rodríguez Arteche, I. y Martínez Aznar, M.M (2018). Evaluación de una propuesta para la formación inicial del profesorado de Física y Química a través del cambio en las creencias de los participantes. Revista Eureka sobre Enseñanza y Divulgación de las Ciencias, 15(1), 1601-1615.

Rodríguez-Arteche, I. y Martínez-Aznar, M.M. (2016). Introducing inquiry-based methodologies during initial secondary education teacher training using an open-ended problem about chemical change. Journal of Chemical Education, 93(9), 1528-1535.

Segers, M. y Tillema, H. (2011). How do Dutch secondary teachers and students perceive the purpose of assements? Studies in Educational Education (special issue), 37, 49-54.

Serrano, R., Pontes, A. y Pérez, E. (2019). Beliefs on the Teacher Professionalism and Teaching Models in Initial Teacher Education. Revista Brasileira de Educação. 24, 1-24.

Shulman, L.S. (1986). Those who understand: knowledge growth in teaching. Educational Researcher, 15(2), 4-14.

Smith, D. y Neale, D. (1991). The Construction of Subjet- Matter Knowledge in Primary Science Teaching, en Brophy, J. (ed.). Advances in Research on Teaching. Vol. 2. 187-243. Greenwich, CT: JAI Press.

Solís, E. (2005). Concepciones curriculares del profesorado de física y química en formación inicial (Tesis Doctoral). Universidad de Sevilla.

Solís, E., Martín, R., Rivero, A. y Porlán, R. (2013). Expectativas y concepciones de los estudiantes del MAES en la especialidad de Ciencias. Revista Eureka sobre Enseñanza y Divulgación de las Ciencias, 10(Extra), 496-513.

Spear, M.G. (1984). Sex bias in science teachers' ratings of work and pupil characteristics, European Journal of Science Education, 6(4), 369-377.

Subramaniam, K. (2013). "Minority Pre-service Teachers' Conceptions of Teaching Science: Sources of science teaching strategies". Research in Science Education 43(2), 687-709.

Subramaniam, K. (2014). Student teachers' conceptions of teaching biology. Journal of Biological Education, 48(2), 91-97.

Tigchelaar, A., J., Vermunt, y Brouwer, N. (2012). Patterns of Development in Second Career Teachers' Conceptions of Learning. Teaching and Teacher Education, 28, 1163-1174.

Turpo-Gebera, O.W. (2011). Concepciones y prácticas evaluativas de los docentes del área curricular de ciencias en las instituciones de enseñanza públicas de educación secundaria. Revista Iberoamericana de Evaluación Educativa, 4(2), 213-233.

Virtanen, V. y Lindblom-Ylänne, S. (2010). University students' and teachers' conceptions of teaching and learning in the biosciences. Instructional Science, 38, 355-370.

Wang, J. R., Kao, H. L., \& Lin, S. W. (2010). Preservice teachers' initial conceptions about assessment of science learning: The coherence with their views of learning science. Teaching and Teacher Education, 26(3), 522-529.

Yakar, Z. \& Turgut, D. (2017). Effectiveness of Lesson Study Approach on Preservice Science Teachers' Beliefs. International Education Studies, 10(6), 36-43. 